Original article

\title{
The Value of Exercise Treadmill Test in Evaluation of Coronary Artery Disease
}

\author{
Lili Mao ${ }^{2}$, Xueqi Li ${ }^{1}$, Lihua Zhong ${ }^{1}$ and Shipeng Wei ${ }^{1,3}$ \\ ${ }^{1}$ Department of Cardiology, the 4th Clinical Hospital of Harbin Medical University, Harbin, 150001, People's Republic of China. \\ ${ }^{2}$ Department of Infectious Diseases, the 1st Affiliated Hospital of Harbin Medical University, Harbin, 150010 \\ ${ }^{3}$ Department of cell, developmental \& integrative biology, University of Alabama at Birmingham, Birmingham, AL 35294
}

Received 14 July 2012, Accepted 24 August 2012.

(C) 2012, Mao L., Li X., Zhong L., Wei S.

Abstract: Objective: To evaluate the value of Exercise treadmill test (ETT) in the diagnosis of coronary artery disease (CAD). Methods: Here

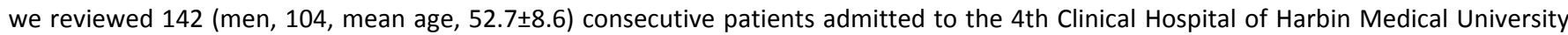
for suspicion of CAD and they underwent ETT before coronary angiography (CAG). Patients were divided into four groups to see the sensitivity of different ETT criteria: group I: ETT negative, group II: ETT positive (ST-segment depression $\geq 0.1 \mathrm{mv}$ for more than 2 minutes), group III: ETT positive (exercise induced chest pain), group IV: ETT positive (exercise induced chest pain and ST-segment depression). The severity of coronary artery stenosis was assessed by CAG, only those with $>50 \%$ of narrowing in at least one of the three major arteries or their first-order branches was considered CAG positive. Results: The false negative rate was $30.6 \%$ and the accuracy rate was $69.4 \%$ in group I. In group II, III and IV, the accuracy rates and false positive rates were $53.7 \%$ and $46.3 \%, 78.6 \%$ and $21.4 \%, 86.7 \%$ and $13.3 \%$, respectively, $(p<0.05)$. Furthermore, we analyzed the data of male patients in each group and the accuracy rates were $67.9 \%, 60.7 \%$, $76.9 \%, 100 \%$, respectively, $(p<0.05)$. Multivariate logistic regression results showed that ST-segment depression together with exercise induced chest pain were the most related factors in CAD diagnosis. Conclusion: ST-segment depression and exercise induced chest pain are the strongest factors in CAD diagnosis. Chest pain combined with ST-segment depression had a much higher accuracy rate than ST-segment depression alone.

Keywords: exercise treadmill test (ETT), coronary artery disease (CAD), chest pain, coronary angiography (CAG).

Cite as Mao L, Li X, Zhong L, Wei S. The Value of Exercise Treadmill Test in Evaluation of Coronary Artery Disease. Russian Open Medical Journal 2012; 1: 0306.

Correspondence to Dr. Shipeng Wei. Address: Department of Cardiology, The Fourth Clinial Hospital of Harbin Medical University, 37 Yiyuan Str., Nangang District, Harbin 15001, China. E-mail: shipengwei@yahoo.com.

\section{Introduction}

Exercise treadmill test (ETT) is one of the most used means in evaluation of coronary artery disease (CAD). Since the first medical treadmill designed to diagnose heart and lung disease was invented by Dr. Robert Bruce and Wayne Quinton at the University of Washington in 1952, it became the golden criteria in diagnosing CAD till coronary angiography (CAG) came out. However, it is still a very easy and comprehensive way in diagnosis of CAD. The position of ETT is going down these years by its high false positive and negative rates [1]. People are looking for more parameters that ETT can provide to increase its accuracy rate, like ST/HR slope, Duke treadmill score, QT dispersion and so on [2-4]. ST-segment depression and chest pain as the classic criteria for CAD diagnosis are well known and accepted. However, each has their own limitations. ST-segment depression is often modified by some other factors, like blood pressure, cigarettes smoking, serum cholesterol level, etc [5]. Chest pain is a subjective complaint which maybe obscured by patients own feelings. Based on what mentioned above, the false positive and negative data is inevitable. Here we questioned which one of the criteria is better in CAD diagnosis, ST-segment depression or chest pain? We reviewed the clinical records of 142 patients who received ETT before CAG to see the coincidence rate of these two examinations. Multivariate logistic regression analysis revealed that both exercise induced ST-segment depression and chest pain were strong factors in CAD diagnosis. Interestingly, we found that chest pain, which is considered subjective, seems more reliable than the objective one, ST-segment depression. The usefulness of ETT in male patients is much greater than that in female ones.

\section{Methods \\ Study population}

300 medical records of consecutive patients with normal resting electrocardiogram (ECG) who were admitted to our hospital and received coronary angiography between 2005 and 2009 were reviewed and 142 of them (men 104, mean age, 52.7 \pm 8.6 ) with complete data were enrolled. Patients were assigned into four groups: group I: ETT negative, group II: ETT positive (exercise induced ST-segment depression $\geq 0.1 \mathrm{mv}$ for more than 2 minutes), group III: ETT positive (exercise induced chest pain), group IV: ETT positive (exercise induced chest pain and ST-segment depression). On further study, they were divided into two groups by gender ( $G M=$ male group and $G F=f$ emale group) to see the differences on 
Cardiology

ETT results between male and female patients. The data of male patients in each group was extracted and analyzed to calculate the accuracy rates among the four groups, too.

\section{Information of Physical Examination}

Blood pressure including systolic and diastolic pressure and heart rate were recorded on admission. The histories of other diseases, like hypertension, diabetes were recorded according to the narration of the patients.

\section{Exercise Treadmill Test}

Patients performed the exercise treadmill tests on a motor-driven treadmill (En Mill, Enraf Nonius BV, Rotterdam, The Netherlands), programmed for increases in angle of inclination and speed for the original Bruce protocol [6]. The target heart rate was calculated by the formula: target heart rate $=(220$-age $) \times 0.85$. The 12 -lead electrocardiogram (ECG) was recorded before ETT and the realtime ECG and blood pressure were monitored during the whole process of ETT. ECGs were recorded every 2 minutes since the exercise stopped till 10 minutes. The main criteria for stopping the exercise were: reaching the target heart rate, reaching $85 \%$ of the target heart rate, unreleasing chest pain or failing for fatigue.

Results meet one of the following criteria were considered positive: chest pain; visually horizontal or down-slope ST-segment depression $\geq 0.1 \mathrm{~mm} 80 \mathrm{~ms}$ after J junction for 2 minutes; STsegment arcade elevation $\geq 0.2 \mathrm{~mm}$ for 1 minute or severe arrhythmias.

\section{Coronary angiography}

Coronary angiography was performed with the standard Judkins approach in all patients who gave written consent as previously discrived [7]. A total of 5-6 views or left coronary artery and three views of right coronary artery were taken. The coronary angiography was reviewed by two independent experienced cardiologists unaware of the patient's general data. Luminal stenosis was calculated as the percentage of diameter reduction in diseased segment compared to the proximal disease-free reference segment. More than $50 \%$ of stenosis in either one of three major coronary arteries including the left anterior descending artery (LAD), left circumflex artery (LCX), and right coronary artery (RCA) or their first-order branches was considered as significant for CAD.

\section{Left Ventricular Ejection Fraction}

The Vivid 7 color Doppler ultrasonic purchased from GE Healthcare (Shanghai, China) was applied for the evaluation of cardial function as previously described [8]. The left ventricular endocardial borders were recorded from the projected left ventriculogram in end systole and diastole. Ejection fraction of patients was confirmed by echocardiographic assessment.

\section{Statistics Analysis}

SPSS 17.0 software was used to analyze the data, it was shown as Mean \pm S.D. A one-way ANOVA is used to determine differences among the group means. Differences at $p<0.05$ were considered significant. The accuracy rate among the groups was analyzed with the Chi-square test. Univariate and multivariate logistic regression analysis were applied to study the relationship of multiple risk factors with CAD diagnosis.
Table 1. General characteristics of the patients

\begin{tabular}{|c|c|c|c|c|}
\hline Variables & $\begin{array}{l}\text { Group I } \\
(n=72)\end{array}$ & $\begin{array}{l}\text { Group II } \\
(n=41)\end{array}$ & $\begin{array}{c}\text { Group III } \\
(n=14)\end{array}$ & $\begin{array}{c}\text { Group IV } \\
(n=15)\end{array}$ \\
\hline Age (years) & $52.5 \pm 8.8$ & $53.6 \pm 8.6$ & $51.3 \pm 8.9$ & $54.0 \pm 7.3$ \\
\hline Male (n) & 54 & 27 & 13 & 10 \\
\hline $\begin{array}{l}\text { Hypertension } \\
\text { history (n) }\end{array}$ & 36 & 19 & 6 & 10 \\
\hline $\begin{array}{l}\text { Diabetes history } \\
\text { (n) }\end{array}$ & 11 & 6 & 1 & 6 \\
\hline HR (beats/min) & $73.0 \pm 8.1$ & $73.7 \pm 7.7$ & $75.0 \pm 6.7$ & $72.0 \pm 7.5$ \\
\hline $\mathrm{SBP}(\mathrm{mmHg})$ & $129.7 \pm 17.5$ & $132.4 \pm 13.7$ & $138.9 \pm 15.3$ & $131.1 \pm 17.8$ \\
\hline DBP $(\mathrm{mmHg})$ & $81.2 \pm 11.5$ & $81.7 \pm 8.8$ & $87.9 \pm 8.9$ & $78.3 \pm 10.9$ \\
\hline $\mathrm{TC}(\mathrm{mmol} / \mathrm{L})$ & $4.91 \pm 1.09$ & $4.65 \pm 0.95$ & $4.96 \pm 0.80$ & $4.56 \pm 0.76$ \\
\hline TG (mmol/L) & $2.10 \pm 1.38$ & $1.82 \pm 0.84$ & $2.07 \pm 1.00$ & $2.24 \pm 1.21$ \\
\hline $\mathrm{HDL}-\mathrm{C}$ (mmol/L) & $1.13 \pm 0.31$ & $1.09 \pm 0.17$ & $1.03 \pm 0.21$ & $1.01 \pm 0.14$ \\
\hline LDL-C (mmol/L) & $2.96 \pm 0.85$ & $2.93 \pm 0.81$ & $3.15 \pm 0.72$ & $2.73 \pm 0.62$ \\
\hline $\mathrm{EF}(\%)$ & $63.6 \pm 4.2$ & $63.2 \pm 6.1$ & $63.7 \pm 3.3$ & $65.3 \pm 4.9$ \\
\hline
\end{tabular}

HR - heart rate, SBP - systolic blood pressure, DBP - diastolic blood pressure, TC - total cholesterol, TG - triglyceride, HDL-C - high-density lipoprotein C, LDL-C - low-density lipoprotein C, EF - eject fraction.

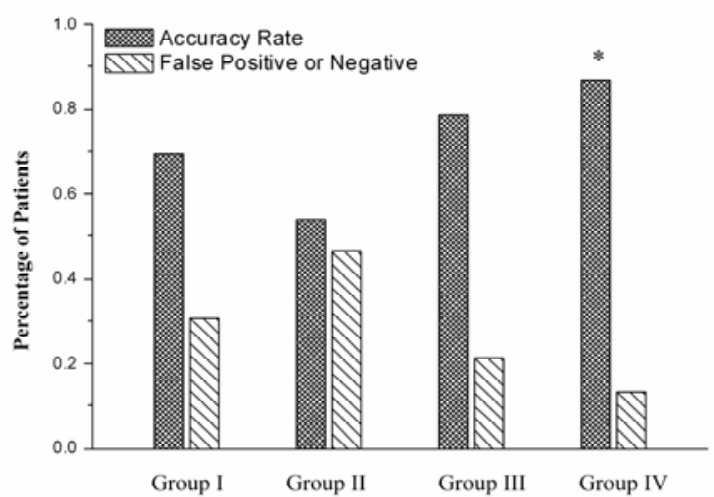

Figure 1. The accuracy rate of ETT in all patients.

Accuracy rates (dense bar) and false positive or negative rates (sparse bar) of each group were shown as group I: $69.4 \%$ and $30.6 \%$, group II: $53.7 \%$ and $46.3 \%$, group III: $78.6 \%$ and $21.4 \%$, group IV: $86.7 \%$ and $13.3 \%$, respectively. Compare to group II, the accuracy rate was higher in group IV, $p<0.05$.

\section{Results}

\section{General characteristics of the patients}

The general information of the patients was described in table 1 . There were 72 patients in group I, 41 in group II, 14 in group III and 15 in group IV. Age, gender, hypertension history, diabetes history, blood lipid level and ejection fraction were recorded and shown in table 1.

\section{Patients with chest pain and ST-segment depression during ETT} are more likely to have CAD

50 out of 72 patients in group I who were not diagnosed CAD by ETT were proved to be CAD free by CAG, while the other 22 were CAD patients, which was shown in figure 1 . The accuracy rate and false negative rate were $69.4 \%$ and $30.6 \%$, respectively. In group III, patients having chest pain alone during ETT, the ETT results were coincident with CAG in 11 patients and the other 3 were considered false positive. The accuracy rate and false positive rate were $78.6 \%$ and $21.4 \%$, respectively. Compare to those in group II, 
$53.7 \%$ and $46.3 \%$, chest pain alone had a higher diagnosis rate than ST-segment depression alone. What's more, in group IV, the accuracy rate was $86.7 \%$, compare to ST-segment depression alone, $53.7 \%, p<0.05$. Also in male patients (general information was shown in table 2), the accuracy rates of these four groups were still different. As shown in figure 2, group III and IV had much higher accuracy rates than that of group II, $76.9 \%$ vs. $60.7 \%$ and $100 \%$ vs. $60.7 \%, p<0.05$. In other words, patients with chest pain and ST-segment depression during ETT are more likely to have CAD.

Table 2. Summary of general information of male patients

\begin{tabular}{lcccc}
\hline \multicolumn{1}{c}{ Variables } & $\begin{array}{c}\text { Group I } \\
(n=54)\end{array}$ & $\begin{array}{c}\text { Group II } \\
(n=27)\end{array}$ & $\begin{array}{c}\text { Group III } \\
(n=13)\end{array}$ & $\begin{array}{c}\text { Group IV } \\
(n=10)\end{array}$ \\
\hline Age (years) & $51.4 \pm 8.4$ & $52.7 \pm 9.0$ & $50.7 \pm 8.9$ & $52.5 \pm 7.0$ \\
Hypertension history (N) & 25 & 14 & 5 & 4 \\
Diabetes history (N) & 9 & 4 & 1 & 5 \\
EF (\%) & $63.4 \pm 3.8$ & $62.5 \pm 7.0$ & $63.6 \pm 3.4$ & $65.1 \pm 5.6$ \\
\hline
\end{tabular}

$\mathrm{EF}$ - eject fraction.

Table 3. The contribution of different factors in CAD diagnosis Univariate analysis

\begin{tabular}{|c|c|c|}
\hline Variables & $F$ & Pvalue \\
\hline Age & 0.680 & 0.412 \\
\hline Diabetes history & 2.872 & 0.093 \\
\hline Hypercholesterolemia & 0.001 & 0.976 \\
\hline Exercise test induced chest pain & 5.075 & 0.027 \\
\hline ST-segment depression & 4.893 & 0.029 \\
\hline Target heart rate & 0.003 & 0.954 \\
\hline Angina history & 3.039 & 0.084 \\
\hline
\end{tabular}

Multivariate analysis

\begin{tabular}{|c|c|c|c|}
\hline Variables & $O R$ & $95 \% \mathrm{Cl}$ & Pvalue \\
\hline ST-segment depression & 3.129 & $1.436-6.822$ & 0.004 \\
\hline Exercise test induced chest pain & 1.872 & $\begin{array}{c}1.724- \\
24.504\end{array}$ & 0.006 \\
\hline
\end{tabular}

\section{Male patients have a higher accuracy rate than female individuals}

The patients were further divided into 2 groups by gender. 73 out of 104 male patients were detected CAD both by ETT and CAG, the accuracy rate was $70.2 \%$ which was much higher than that $(50.0 \%)$ of the female patients, $p<0.05$ as shown in figure 3 .

\section{Chest pain and ST-segment depression during ETT are the strongest factors in diagnosis of CAD}

The relationship of different factors with CAD diagnosis was studied by univariate and multivariate logistic regression analysis. As shown in table 3 , the relationship of the most possible related factors, age $(<55=0, \geq 55=1)$, diabetes (no $=0$, yes $=1$ ), hypercholesterolemia (no=0, yes=1), ST-segment depression $(<0.1 \mathrm{~mm}=0, \geq 0.1 \mathrm{~mm}=1, \geq 0.2 \mathrm{~mm}=2)$, exercise induced chest pain (no $=0$, yes $=1$ ), fulfilled heart rate $(<100 \mathrm{bpm}=1,100-129=2,130$ $159=3,160-189=4,189-220=5$ ), angina history (non-cardiac chest pain $=0$, probable chest pain $=1$, typical angina $=2$ ) were analyzed by univariate logistic regression. Exercise induced ST-segment depression and chest pain were shown associated with CAD diagnosis, $p=0.029$ and 0.027 , respectively. In the following multivariate logistic regression model, both of them still showed significant relationship with CAD diagnosis, $\mathrm{p}=0.006$ and 0.004 respectively.

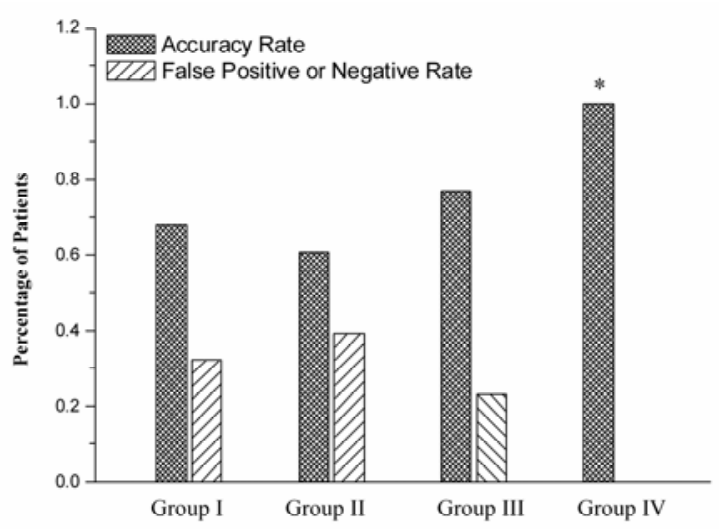

Figure 2. The accuracy rate of ETT in male patients.

Accuracy rates (dense bar) and false positive or negative rates (sparse bar) of the male patients among each group were shown, compare to STsegment depression alone (group II), ST-segment depression plus chest pain (group IV) showed a higher accuracy rate, $100 \%$ vs. $60.7 \%, p<0.05$.

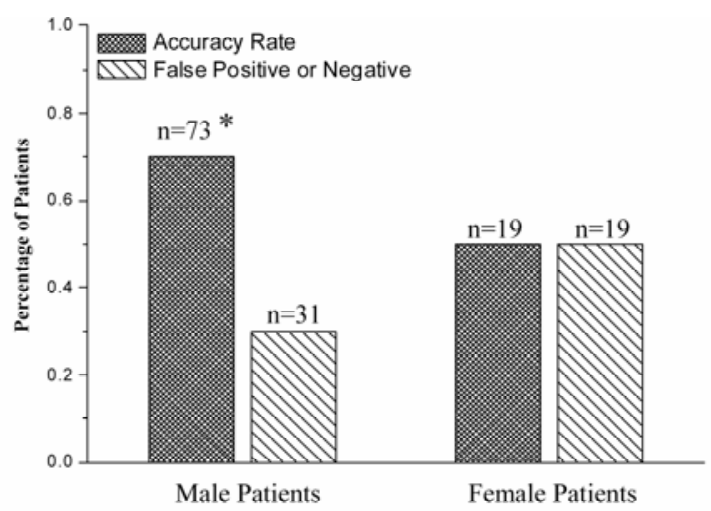

Figure 3. The difference of accuracy rate of ETT between male and female patients.

The difference of accuracy rates between male and female patients was shown in the panel (dense bar), $70.2 \%$ vs. $50.0 \%, p<0.05$.

\section{Discussion}

Several conclusions were made based on the present study: 1) exercise induced ST-segment depression and chest pain are the strongest predictive factors in CAD diagnosis. 2) Chest pain combined with ST-segment depression had a much higher accuracy rate than ST-segment depression alone. 3) ETT is more valuable in CAD diagnosis in male patients than in those of female.

\section{ST-segment depression and chest pain}

As the long and classically used technique, ETT has been in predominant position for years until CAG was brought up by which ETT was greatly challenged. It is no doubt that something old will 
be taken placed by something new. However, nowadays ETT still has considerable value in diagnosis of CAD. The other is that ETT is much easier for the patients to accept because it is noninvasive and easily applicable. However, we argue why the false negative and positive rates are so high? The most direct reason is the criteria for CAD diagnosis. As we know, the golden criteria for $C A D$ diagnosis by ETT are: chest pain and visualized horizontal or downslope ST-segment depression $\geq 0.1 \mathrm{~mm} 80 \mathrm{~ms}$ after $\mathrm{J}$ junction for 2 minutes. They were shown strong predictors for the mortality of male patients in long-time follow-up [9]. As an objective parameter, ST-segment depression was thought more reliable than the subjective feeling, chest pain. However, it is not the case from the view of our data. The accuracy rate of the patients having chest pain alone is $78.6 \%$, which is much higher than the $53.7 \%$ among patients with ST-segment depression alone, though it didn't show significant difference due to sample size. If chest pain was combined with ST-segment depression, the accuracy rate was increased to $86.7 \%$ which was significant compared to ST-segment depression alone. Taking gender for consideration, we further investigated the accuracy rate in male patients as shown in table 3. Accuracy rates in male patients with chest pain alone or both chest pain and ST-segment depression were still higher than that of STsegment depression alone, $76.9 \%$ vs. $60.7 \%$ and $100 \%$ vs. $60.7 \%$, $\mathrm{p}<0.05$, which was similar to what has been reported by Starling and his colleagues [10]. All these information indicates that chest pain accompanied by ST-segment depression is a better parameter than ST-segment depression alone.

\section{The value of ETT in diagnosis of CAD in male and female patients}

The difference of the value of ETT in diagnosis of CAD in male and female patients was well known [11]. The accuracy rate of ETT in female patients was low. Since women had a much higher mortality than men [12], ETT was not considered a sensitive way in diagnosis of CAD in female patients. In our study, we found the same phenomena that the accuracy rate in male group was much higher than that of female group, $70.2 \%$ vs. $50.0 \%$. This number is pretty close to what has been reported in the literature, however, ETT is still the first recommendation in evaluating the severity of CAD in female patients according to the guideline [13]. Nevertheless, ETT is obviously not enough for them, so some people insist that these patients should take multi-detector computed tomography (MDCT) for further examination [14]. As for the doctors, some of them may suggest the female patients to take MDCT in the first place, but it is not right in terms of saving medical resources. So the traditional ETT may not work for the female patients, while some modified methods might be evaluated, such like spectral analysis of heart rate variability, which was reported by V.I. Gridnev et al [15].

\section{ETT score system for diagnosis of CAD}

Since the false positive and negative rates are high, some score systems were brought up in order to raise the accuracy rate of ETT. Vondi, et al brought up with a score system to evaluate STsegment depression and chest pain for diagnosis of CAD [6]. Although this system has an over $80 \%$ of accuracy rate, it still puts ST-segment depression in more important position over chest pain. We calculated the scores based on this system and our data indicated that the scores in ETT positive groups were much higher than that of ETT negative group, $37.2 \pm 7.0$ vs. $56.8 \pm 7.5,55.1 \pm 11.8$ and $64.3 \pm 9.4$ (data not shown). Obviously ST-segment depression had a more important position in this system, but the scores in the patients of chest pain alone and ST-segment depression alone are almost equal. From the multivariate logistic regression analysis, STsegment depression and chest pain were almost equally important. Of course, it may not be convincing due to the small sample size.

Another old score system, Duke treadmill score, seemed to well balance the ST-segment depression and chest pain [3]. However, other clinical informations, like age, lipid level or heart rate are not included. Other means, such as ST/HR slope, QT dispersion were reported to be more accurate than ST-segment depression alone $[2,4]$. So probably a more comprehensive system should be developed in assessing the results of ETT.

\section{Conclusions}

Exercise induced ST-segment depression and chest pain are the strongest factors in CAD diagnosis. Chest pain combined with STsegment depression had a much higher accuracy rate than STsegment depression alone. It also shows that ETT is more valuable in CAD diagnosis in male patients than in those of female.

\section{Acknowledgements}

This work was supported by the educational grant for technology of Heilongjiang Province (grant number: 11541125) to Dr. Lihua Zhong. Dr. Jiwei Du, the director of medical record library of the fourth clinical hospital of Harbin Medical University helped us with the cases review.

\section{Conflict of interest}

All authors do not have conflict of interest.

\section{Reference}

1. Zhang S-L, Jiang $\mathrm{Y}, \mathrm{Xu} \mathrm{H}-\mathrm{M}, \mathrm{Qju} \mathrm{H}-\mathrm{X}$. A comparative study on treadmill exercise test and coronary angiography in the diagnosis of coronary artery di-sease: report of 267 cases. Journal of the Fourth Military Medical University 2007; 28(21).

2. Kronander H, Fischer-Colbrie W, Nowak J, Brodin LA, Elmqvist H. Diagnostic performance and partition values of exercise electrocardiographic variables in the detection of coronary artery disease--improved accuracy by using ST/HR hysteresis. Clin Physiol Funct Imaging 2010; 30(2): 98-106 (doi: 10.1111/j.1475097X.2009.00909.x) (PMID: 19919616)

3. Lee DH, Jeon HK, Park HJ, Shin WS, Lee SW, Youn HJ, et al. Change in ischemia-modified albumin and its clinical significance during exercise stress testing. Circ J 2010; 74(3): 484-489 (PMID: 20057159) (doi: 10.1253/circj.CJ-09-0581).

4. Takase B, Masaki N, Hattori H, Ishihara M, Kurita A. Usefulness of automatic QT dispersion measurement for detecting exercise-induced myocardial ischemia. Anadolu Kardiyol Derg 2009; 9(3): 189-195 (PMID: 19520652)

5. Zheng $\mathrm{YX}$, Lin $\mathrm{H}$. The Analysis of the Factors Influencing the Accuracy of Treadmill Electrocardiogram Test. Journal of Practical Medical Techniques 2007: 14(17) (doi: CNKI:SUN:SYYJ.0.2007-17-008).

6. Van der Cammen-van Zijp MH, ljsselstijn $\mathrm{H}$, Takken $\mathrm{T}$, Willemsen SP, Tibboel D, et al. Exercise testing of pre-school children using the Bruce treadmill protocol: new reference values. Eur J Appl Physiol 2010; 108(2): 393-399 (PMID: 19821120) (doi: 10.1007/s00421-009-1236-x).

7. Wei S, Zhong L, Chen S, Li X. The status of coronary artery lesions in patients with conduction disturbance. J Cardiovasc Med (Hagerstown) 2011; 12(10): 709-713 (doi: 10.2459/JCM.0b013e328349187c) (PMID: 21709574).

8. Gao C, Zhong L, Gao Y, Li X, Zhang M, Wei S. Cystatin C levels are associated with the prognosis of systolic heart failure patients. Arch Cardiovasc Dis 2011; 104(11): 565-571 (PMID: 22117908) (doi: 10.1016/j.acvd.2011.08.003) 
9. Prakash M, Myers J, Froelicher VF, Marcus R, Do D, et al. Clinical and exercise test predictors of all-cause mortality: results from $>6,000$ consecutive referred male patients. Chest 2001; 120(3): 1003-1013 (doi:10.1378/chest.120.3.1003).

10. Starling MR, Kennedy GT, Crawford MH, O'Rourke RA. Comparative predictive value of ST-segment depression or angina during early and repeat postinfarction exercise tests. Chest 1984; 86(6): 845-849 (PMID: 6499545) (doi:10.1378/chest.86.6.845).

11. Shah SF, Meo SA. Usefulness of standard treadmill stress testing in women. J Pak Med Assoc 2009; 59(4): 197-200 (PMID: 19402276).

12. Shaw LJ, Bugiardini R, Merz CN. Women and ischemic heart disease: evolving knowledge. J Am Coll Cardiol 2009; 54(17): 1561-1575 (PMID: 19833255) (doi:10.1016/j.jacc.2009.04.098).

13. Gibbons RJ, Balady GJ, Bricker JT, Chaitman BR, Fletcher GF, et al. ACC/AHA 2002 guideline update for exercise testing: summary article: a report of the American College of Cardiology/American Heart Association Task Force on Practice Guidelines (Committee to Update the 1997 Exercise Testing Guidelines). Circulation 2002; 106: 18831892 (PMID: 12392846) (doi: 10.1161/01.CIR.0000034670.06526.15).

14. Starr H, Powers L, Safranek S. Clinical Inquiries: What is the best noninvasive diagnostic test for women with suspected CAD? J Fam Pract 2010; 59(9): 534-535 (PMID: 20824233).

15. Gridnev VI, Kiselev AR, Posnenkova OM, Shvartz VA. Using of spectral analysis of heart rate variability for increasing reliability of bicycle ergometry results. Health 2011; 3(8): 477-481 (doi: 10.4236/health.2011.38078)

\section{Authors:}

Lili Mao - B.S., Senior Researcher, Department of Infectious Diseases, the 1st Affiliated Hospital of Harbin Medical University, Harbin, 150010;

Xueqi Li - MD, Professor, Department of Cardiology, The 4th Clinical Hospital of Harbin Medical University, Harbin, 150001, People's Republic of China;

Lihua Zhong - MD, Associate Professor, Department of Cardiology, The 4th Clinical Hospital of Harbin Medical University, Harbin, 150001, People's Republic of China;

Shipeng Wei - (1) MD, Assistant Professor, Department of Cardiology, The 4th Clinical Hospital of Harbin Medical University, Harbin, 150001, People's Republic of China; (2) Research Associate, Department of cell, developmental \& integrative biology, University of Alabama at Birmingham, Birmingham, AL 35294. 